\title{
Automatic Analysis of Normal Brain Dissymmetry of Males and Females in MR Images
}

\author{
Sylvain Prima ${ }^{1}$, Jean-Philippe Thirion ${ }^{2}$, Gérard Subsol $^{1}$, and Neil Roberts ${ }^{3}$ \\ 1 INRIA Sophia Antipolis, EPIDAURE Project, France \\ Sylvain.Prima@sophia.inria.fr \\ 2 Focus Imaging SA, Sophia Antipolis, France \\ 3 MARIARC, University of Liverpool, U.K.
}

\begin{abstract}
In this paper, we present a statistical analysis of the normal dissymmetry in brain MR images. Our method consists in: computing a dense 3D field characterizing the dissymmetry of a subject, analysing it with an operator (relative volume variation), computing $3 \mathrm{D}$ statistical maps from populations of subjects (learning series) and extracting interesting regions. The results are then tested on an independent validation series. We use a database of 36 normal young subjects, 18 males and 18 females, strongly right-handed. We show that the brain hemispheres can be reliably discriminated thanks to a consistent size difference in the white matter of the temporal and frontal lobes, with a very high level of confidence $(36 / 36, \alpha$-value $=0.000001)$. We also exhibit a difference of brain dissymmetry with respect to sex.
\end{abstract}

\section{Introduction}

In [10], we presented a general method to study the dissymmetry of anatomical structures such as the human brain. This work was initially motivated by clinical studies assuming a connection between diseases such as schizophrenia and abnormalities in brain symmetry [1]. In this work, we computed at each voxel of a 3D MR brain image a vector characteristic of the dissymmetry and we obtained a dense $3 \mathrm{D}$ vector field $F$ called the "dissymmetry field" of the image. This was then analysed with the operator $\|F\| \operatorname{div}(F)$ that gives a "dissymmetry image" enhancing the differences between "symmetric" anatomical structures in the hemispheres, such as ventricles, temporal lobes, etc. From images computed using several subjects, we deduced 3D statistical maps to detect significantly dissymmetrical locations in a given population, or to compare the dissymmetry between 2 populations, or between one subject and a population.

In this paper, we present a more detailed statistical analysis of the dissymmetry field and we apply the whole method to large databases of subjects. In section 2 , we recall the general scheme of the method and introduce a new $3 \mathrm{D}$ vector field operator, the relative volume variation measure. In section 3 , we propose a further step in the statistical analysis to validate the results: after computing statistical maps from a learning series of subjects, we automatically extract interesting areas that we investigate in a different validation series. In section 
4, we present new experiments on a large database of subjects: after analysing the "normal" dissymmetry in one population (normal right-handed young males, normal right-handed young males plus females) we compare brain dissymmetry between males and females.

\section{Description of the general scheme}

\subsection{General presentation}

The gross symmetry of the human brain is very well known by clinicians. The cortical surface of the brain, as well as the substructures such as the ventricles, the hippocampi, the white and grey matters, etc., are mainly symmetrical. Some subtle differences can be noted with a closer visual inspection, showing morphometrical (e.g., the size of the ventricles) and morphological (e.g., the number of white matter tracks) differences between homologous structures in both hemispheres. Our goal is to quantify and localise those differences in human brain MR images. Assuming the existence of a symmetry plane in the brain, the basic idea is to compare the initial image with its symmetrical counterpart with respect to this plane. By matching homologous voxels in both hemispheres, a non-rigid registration algorithm gives a $3 \mathrm{D}$ field characterizing the dissymmetry at each voxel; e.g., a perfectly symmetrical brain leads to a null field.

\subsection{Computation of the mid-sagittal plane}

The first step of the algorithm consists in computing the "symmetry plane". The position of the patient's head during the scanning process is variable, and taking the "geometrical" mid-plane of an MR image is only a rough approximation of the sought after "anatomical" mid-plane. The medical description of this plane, usually called the "mid-sagittal plane", relies on an anatomical landmark: the inter-hemispheric fissure of the brain. In [4], the hemisphere boundaries defining this fissure in an MR image are segmented using snakes, and the plane is fitted to a set of control points taken on inter-hemispheric curves. In [3], the plane is estimated from each "mid-sagittal symmetry axis" in all axial (or coronal) slices of an MR or CT image. Each axis maximizes the similarity between the slice and its 2D symmetric reflexion in relation to this axis; the similarity criterion is the $2 \mathrm{D}$ cross-correlation. Some other techniques have been developed for centering functional brain images [5], however the generalization to anatomical modalities seems difficult. Our method, developed in [10] and briefly described in Figure 1 , does not require any preprocessing such as segmentation and relies on the whole volume of the MR image, without any anatomical knowledge; it computes directly the 3D plane minimizing a least-squares criterion. Moreover, it can be generalized to realign any approximately symmetrical object.

In the second step, we compute the rigid transform that realigns automatically the mid-sagittal plane with the mid-plane of the MR image. 


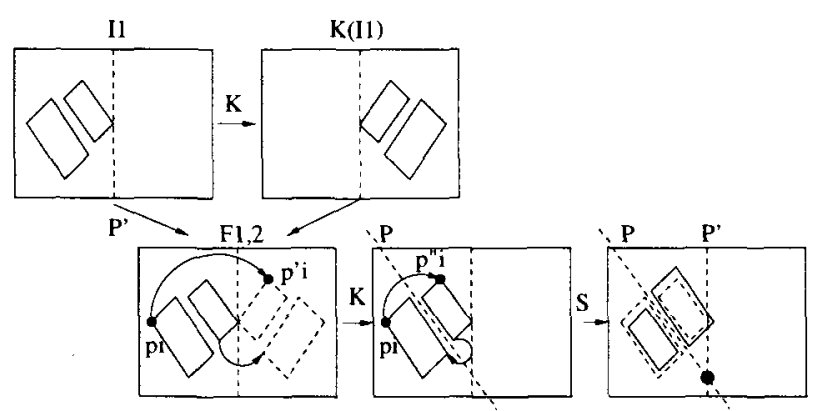

Fig. 1. Overview of the mid-sagittal plane determination: the image $I_{1}$ is transformed into a symmetrical image $K\left(I_{1}\right)$ with respect to any given plane $P^{\prime}$ (in general, the midplane of the image). Then a point to point correspondance $F_{1,2}$ is computed between both images, leading to pairs $\left(p_{i}, p_{i}^{\prime}\right)$. Applying $K$ to the $p_{i}^{\prime}$ points gives couples $\left(p_{i}, p_{i}^{\prime \prime}\right)$ of corresponding points with respect to the mid-sagittal plane $P$, which are used to compute the final symmetry $S$ or equivalently the mid-plane $P$ itself, by minimization of $C=\sum_{i}\left(S\left(p_{i}^{\prime \prime}\right)-p_{i}\right)^{2}=\sum_{i}\left(S \circ K\left(p_{i}^{\prime}\right)-p_{i}\right)^{2}$.

\subsection{Computation of the $3 \mathrm{D}$ dissymmetry image}

Computation of the 3D dissymmetry field. Once the the mid-plane of the MRI is aligned with the mid-sagittal plane, the $3 \mathrm{D}$ deformation field obtained by non-rigid registration between the image and its symmetric is called the "dissymmetry field" of the brain. This 3D field is costly: it is difficult to visualize, to analyse statistically (it requires multivariate analysis tools) and requires a lot of memory. An intuitive idea to extract the most relevant information provided by this field $F$ is to reduce it to a $3 \mathrm{D}$ scalar image, by applying an operator. In [10], we proposed to use the operator $\|F\| \operatorname{div}(F)$, introduced in [9], which gives good results, but whose "physical" meaning is difficult to interpret. Here, we introduce a new operator, $d v / v$, more quantitative and intuitive, that computes the relative volume variation of a set of cells submitted to a deformation field.

Volume variation of a cell. The non-rigid registration provides a $3 \mathrm{D}$ deformation vector for each voxel of the MRI of size $X \times Y \times Z$. We assume that these vectors are applied to the centers of the voxels. Considering the voxel of coordinates $(i, j, k)$, the centers of the voxels $(i, j, k),(i, j+1, k),(i, j, k+1)$, $(i, j+1, k+1),(i+1, j, k),(i+1, j+1, k),(i+1, j, k+1)$, and $(i+1, j+1, k+1)$ define unambiguously the vertices of a cube that we call the cell $(i, j, k)$. We want to compute the volume variation of each cell under the action of the deformation applied to its eight vertices, to build the $3 \mathrm{D}$ image of size $(X-1) \times(Y-1) \times(Z-1)$ where the value of the voxel $(i, j, k)$ is the volume variation of the cell $(i, j, k)$. This first "dissymmetry image", associated with the original MRI, enhances the local differences of size between the hemispheres of the brain.

A priori, each deformed cell is a non-regular hexahedron; we partition each cell into 6 tetrahedra. If $(\boldsymbol{u}, \boldsymbol{v}, \boldsymbol{w})$ defines a positively oriented tetrahedron, its volume is $\frac{1}{6} \operatorname{Det}(\boldsymbol{u}, \boldsymbol{v}, \boldsymbol{w})$. The deformation field, $F$, transforms $\boldsymbol{u}, \boldsymbol{v}, \boldsymbol{w}$ into $F(\boldsymbol{u})$, $F(v), F(\boldsymbol{w})$ respectively; we define the volume of the deformed tetrahedron as 
$\frac{1}{6} \operatorname{Det}(F(\boldsymbol{u}), F(\boldsymbol{v}), F(\boldsymbol{w}))$. If $(F(\boldsymbol{u}), F(\boldsymbol{v}), F(\boldsymbol{w}))$ is no longer direct, the volume of the "transformed tetrahedron" is negative. We define the volume variation of the cell by summing the volume variation of the 6 tetrahedra.

The partition is the same for all cells, so that 2 adjacent cells have a common triangle partition on their common face. We define the volume variation of a whole region of (non-necessarily connected) cells as the sum of each cell volume variation. The result is the actual volume variation of the whole region deformed by the field, which depends only on its boundary displacement. In particular, if the region boudary remains the same, the integration of volume variation over the cells composing this region is null.

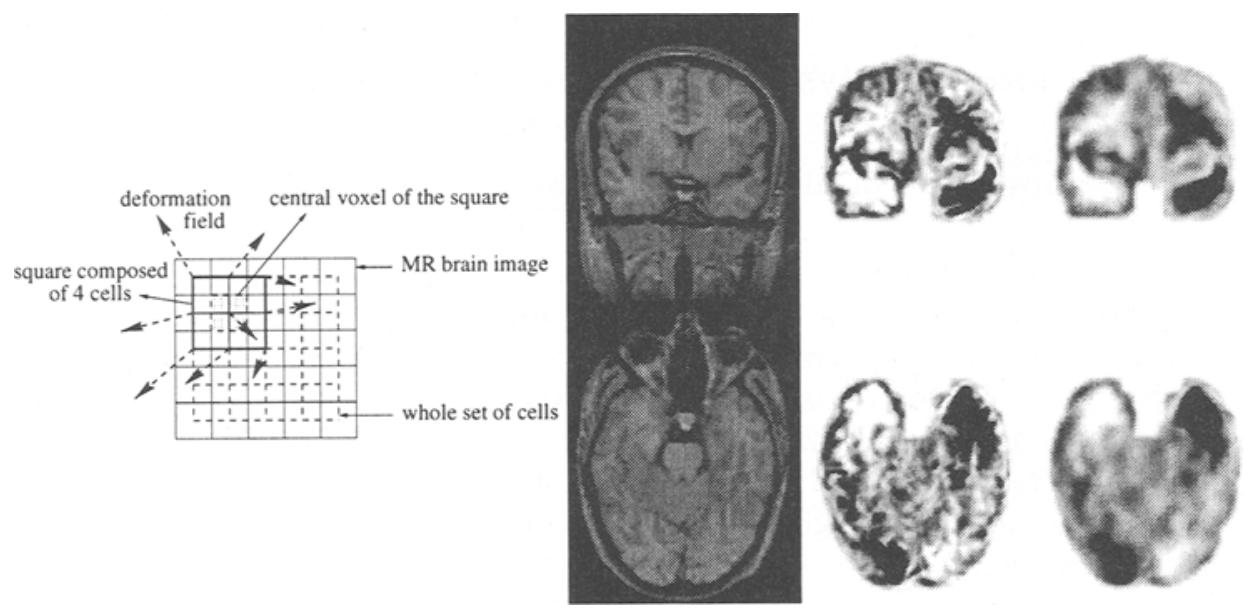

Fig. 2. Left: Integration on a larger cube: here, we present an illustration in the $2 \mathrm{D}$ case with $N=1$. From the original MRI and its associated 2D deformation field, we compute the area variation of all cells, that we integrate on the squares of $(2 N)^{2}=4$ cells and set the normalised value (i.e., area variation divided by 4 ) to the center voxel in the MR image. Right: Comparison between the first dissymmetry image and the smoothed one (coronal and axial views). From left to right: reference subject; first dissymmetry image (white means that the voxel is larger than its correspondent in the other hemisphere); smoothed dissymmetry image, with $N=3$. In accordance with the medical convention, the right side of the subject is shown on the left in the MRI.

Computation of the $d v / v$ image. To compare all the database subjects, we align their dissymmetry images in the same frame (i.e., with respect to a reference subject), with a non-rigid registration algorithm.

This is the third use of this algorithm in the scheme: the method is based on the "demons" algorithm [8], relying on grey values, entirely automatic, and relatively fast: the time computation of the 3D deformation field is about 20 minutes for two $256 \times 256 \times 128 \mathrm{MRI}$ on a DEC Alpha workstation of $400 \mathrm{MHz}$.

Experimentally, we can obtain a rough idea of the accuracy of this registration method: the comparison with other methods gives mutually coherent results 
with an average difference of about 3 voxels [11]. Consequently, it seems inconsistent to perform a voxel-by-voxel statistical analysis on these aligned images. Thus, we perform a linear filtering of these images by taking into account the neighbourhood of each cell. Given an integer $N$, we sum the volume variation of the $(2 N)^{3}$ cells belonging to the cube centered in each voxel (see Figure 2). After division by $(2 N)^{3}$, we obtain a relative volume variation that takes into account the neighbouring values and the new "dissymmetry" $(d v / v)$ image is a smoother version of the first dissymmetry image (see Figure 2). The smoothing of high frequency variations in this one reduces the effects of misregistration, but as $N$ increases, some anatomical details are lost and we get more global dissymmetry measures. Given the estimated accuracy of the registration, we chose $N=3$.

\section{Statistical analysis of dissymmetry}

\subsection{Computation of dissymmetry maps}

Dissymmetry over a given population. We use basic univariate statistical analysis techniques. Given a population of $n$ subjects, their aligned, smoothed dissymmetry images are comparable voxel by voxel. We denote the value of the subject $i$ at a given voxel as $x_{i}$. We assume that $x_{1}, \ldots, x_{n}$ follow a Gaussian law, of unknown mean $\mu_{0}$ and variance $\sigma_{0}^{2} \cdot \mu_{0}=0$ means a perfect symmetry at this voxel. Let $H_{0}: \mu_{0}=0$ and $H_{1}: \mu_{0} \neq 0$ be the null and alternative hypotheses. We compute the $\alpha$-value, giving the probability to be wrong in rejecting the null hypothesis, i.e., in saying that there is a dissymmetry at this voxel:

$$
\alpha=F_{1, n-1}^{-1}\left(T^{2}\right), \quad\left(F_{1, n-1} \text { is a Fisher's law }\right)
$$

where: $T^{2}=\frac{n \mu^{2}}{\sigma}, \mu=\frac{1}{n} \sum_{i=1}^{n} x_{i}$, and $\sigma=\frac{1}{n-1} \sum_{i=1}^{n}\left(x_{i}-\mu\right)^{2}$. Computing this $\alpha$-value for each voxel gives a dense 3D statistical map.

Dissymmetry between 2 given populations. Given two populations of $n_{1}$ and $n_{2}$ subjects, whose dissymmetry images are computed and aligned, we denote the intensity of a given voxel for the first (resp. second) population as $x_{i}^{1}$ (resp., $x_{i}^{2}$ ). We assume that $\left(x_{1}^{1}, \ldots, x_{n_{1}}^{1}\right)$ (resp., $\left(x_{1}^{2}, \ldots, x_{n_{2}}^{2}\right)$ ) follow a Gaussian law, of unknown mean $\mu_{1}$ (resp., $\mu_{2}$ ) and unknown variance $\sigma_{1}^{2}$ (resp., $\sigma_{2}^{2}$ ). $\mu_{1}=\mu_{2}$ means that the dissymmetry of the two populations is the same at this voxel. Let $H_{0}: \mu_{1}=\mu_{2}$ and $H_{1}: \mu_{1} \neq \mu_{2}$ be the null and alternative hypotheses. The probability to be wrong in saying that there is a difference of dissymmetry is:

$$
\alpha=F_{1, n_{1}+n_{2}-2}^{-1}\left(T^{2}\right), \quad\left(F_{1, n_{1}+n_{2}-2} \text { is a Fisher's law }\right)
$$

where: $T^{2}=\frac{n_{1} n_{2}}{\left(n_{1}+n_{2}\right)} \frac{\left(\bar{x}_{2}-\bar{x}_{1}\right)^{2}}{S}, \bar{x}_{1}=\frac{1}{n_{1}} \sum_{i=1}^{n_{1}} x_{i}^{1}, \bar{x}_{2}=\frac{1}{n_{2}} \sum_{i=1}^{n_{2}} x_{i}^{2}$,

and $S=\frac{1}{n_{1}+n_{2}-2}\left[\sum_{i=1}^{n_{1}}\left(x_{i}^{1}-\bar{x}_{1}\right)^{2}+\sum_{i=1}^{n_{2}}\left(x_{i}^{2}-\bar{x}_{2}\right)^{2}\right]$. 


\subsection{Interpretation of the dissymmetry maps}

The first idea is to set a significance level $l$ and to focus on the significant voxels using this level, which is equivalent to computing a two-tailed t-test: we keep voxels whose $\alpha<l$. The dense 3D map is then reduced to a binary image. A typical level for clinical studies is 0.001 , which means, for each voxel there is one-in-a-thousand chance to reject wrongly the null hypothesis. However, the interpretation of these tests can be strongly misleading. Let us suppose that the voxels are uncorrelated; then, the tests are independent, and the probability of wrongly rejecting the null hypothesis for at least one of the $n$ voxels in the image is equal to $1-(1-l)^{n}$. For $l=0.001$, with an image of $n=128^{3} \simeq 2 \times 10^{6}$ voxels, this probability is very close to 1 : we are nearly sure to have false positives between significant voxels, that is voxels for which $\alpha<l$ "by chance".

However, we notice that isolated significant voxels are very rare in this binary map and most of them compose connected clusters of several voxels. This can be partially explained by the spatial correlation between the voxel values of the dissymmetry images; thus, the $n t$-tests we compute (as many as there are voxels in the MR images) are not independent. This correlation can be explained by:

- The anatomical consistency of the dissymmetry: the dissymmetry in substructures, such as the ventricles or the hippocampi, results in a non-null dissymmetry field over their whole volume.

- The iterative Gaussian filtering applied on the deformation field by the demons non-rigid registration algorithm. The smoothing parameter $\sigma$ is constant along iterations and is set to 2.0 .

- The linear filtering of the dissymmetry image, controlled by $N$.

It is then natural to focus on clusters more than on individual voxels in binary maps, and to identify the most "significant" clusters. Such a method is proposed in the software $S P M$ [7], in the field of functional image statistical analysis. A spatially limited cluster is usually considered less "significant" than larger ones, and a further quantitative analysis, with computation of exact probabilities for sets of voxels, can be found in [2]. We propose a simplified method to validate our results. First, we divide the whole database into a learning series and a validation series, and we compute the first dissymmetry image and its smoothed version for each subject. Second, we follow the scheme (see also Figure 3):

- First step: the 3D dense statistical maps are computed on the learning series. Then a significant level is arbitrarily fixed, and the clusters of connected significant voxels are extracted. The 2 hemispheres contain the same information of dissymmetry, and we choose to study one of them only. We identify the $k$ most significant clusters as the $k$ largest ones in this hemisphere.

- Second step: we associate to each of these voxel clusters the corresponding cluster of cells (i.e., the cell $(i, j, k)$ to the voxel $(i, j, k)$ ), whose relative volume variation is computed on the validation series, independently of the learning series. This leads to a series of values that we statistically analyse according to the formulae of section 3.1. 


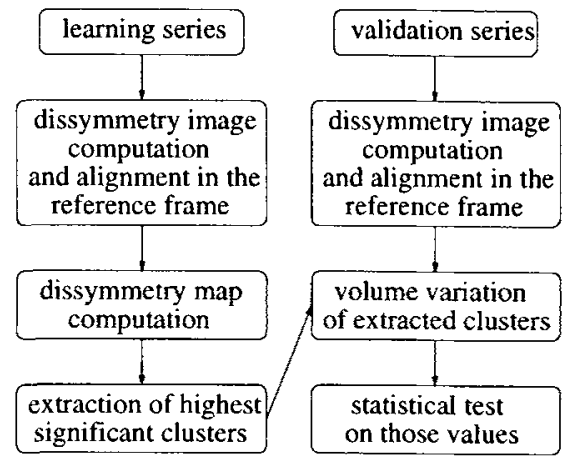

\section{Results}

\subsection{Materials}

Our database is composed of $19 \mathrm{MR}$ brain images of healthy young males and 18 of healthy young females, provided by the MARIARC, University of Liverpool. All of them are strongly right-handed to restrict the possible influence of the laterality on dissymmetry results. One of the males is chosen as the reference subject for all our experiments (perfectly right-handed: laterality coefficient of 100 ). This leaves 36 subjects, divided into the learning series (10 males and 10 females, chosen with the highest laterality coefficient), and the validation series ( 8 males and 8 females). In the following, we perform several experiments.

\subsection{The dissymmetry over the male population}

Figure 4 shows the average dissymmetry and the dissymmetry map for $l=0.001$, computed on the 10 males of the learning series. We reaffirm the preliminary results presented in [10] with a different operator. Two main areas appear in the map: one in the white matter of the temporal lobe, the other in the frontal lobe. Both seem to be significantly larger in the right hemisphere. We focus on these two areas in the left hemisphere (at right on the images, according to the medical convention), and compute their relative volume variation for the 8 males of the validation series. From those values, we deduce the probabilities to be wrong in saying that those areas are dissymmetrical: 0.00041 (temporal lobe) and 0.00044 (frontal lobe). Those values are largely lower than usual significance levels (typically, $l=0.001$ ): this is very conclusive.

One must note another interesting result: in the average dissymmetry image, we recover the torque effect, very well known by clinicians. There is an "inversion" of dissymmetry between the front and the back of the brain. This does not appear in the map: it is not significant over the whole population for $l=0.001$.

\subsection{Comparison males/females}

The comparison of dissymmetry between males and females has been largely studied by biologists. It is widely assumed that language functions are likely to 


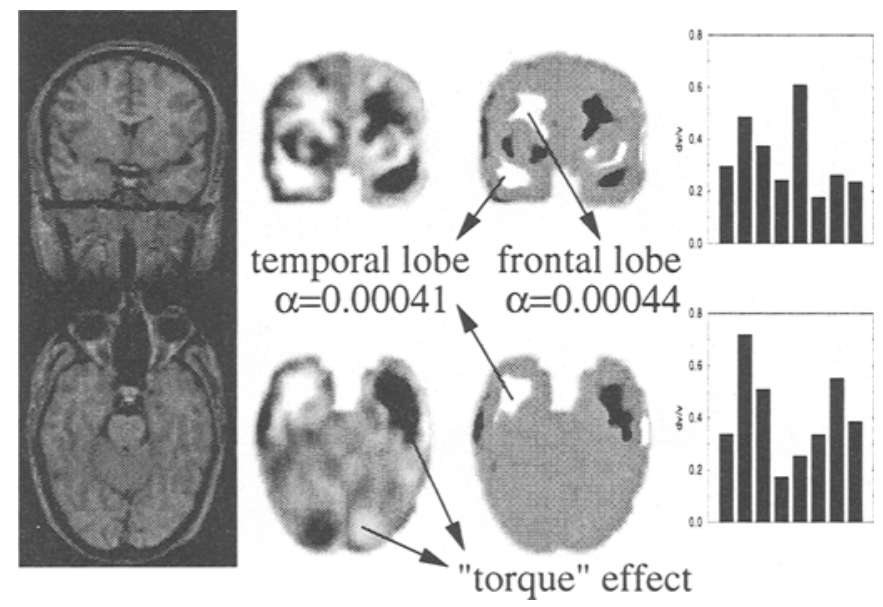

Fig. 4. From left to right: reference subject; average of the $d v / v$ images of the male learning series; statistical map for this series (each voxel is displayed with its $\alpha$-value bearing the sign of the average dissymmetry at this point, $l=0.001) ; d v / v$ measure on the validation series for the 2 most significant clusters in the left hemisphere, located in the frontal (top) and temporal (bottom) lobes.

be more lateralized in males [6]. Males also seem to show greater anatomical dissymmetry than females, e.g., for the Sylvian fissure [12]. Figure 5 shows the average dissymmetry for the 10 males and the 10 females of the learning series and the statistical map for $l=0.001$ comparing these 2 populations. We kept the area in the top of the left hemisphere. The computation of the relative volume variation of this area for the validation series gives 2 series of 8 values. The $\alpha$ value is equal to 0.11 . The area seems to be smaller in the left hemisphere than in the right for the males, and larger for the females. Further experiments on a larger dataset is probably needed to lead to definitive conclusions.

\subsection{The dissymmetry over the whole population}

The comparison between males and females does not lead to a significant difference in the temporal and frontal lobes, which correspond to the largest natural dissymmetry in the male population. The computation of the dissymmetry map for the whole learning series allows one to enhance a "normal dissymmetry" on a wider population. The map, shown in Figure 6 , reveals a large set of voxels, in the same area as for the male only population, but wider, i.e., intuitively more significant. The largest "significant" volume is a "V" shape in the white matter of the cortex, spreading from the frontal to the temporal lobe. Computing the relative volume variation of this " $\mathrm{V}$ " shape in the left hemisphere for the whole database of males and females gives a series of 36 values all superior to 0 , which means that for all subjects, this volume is larger in the right hemisphere than in the left: for our database, we have a criterion allowing to discriminate consistently the left hemisphere from the right one. The computed $\alpha$-value on the validation series is equal to 0.000001 , which confirm these results. 

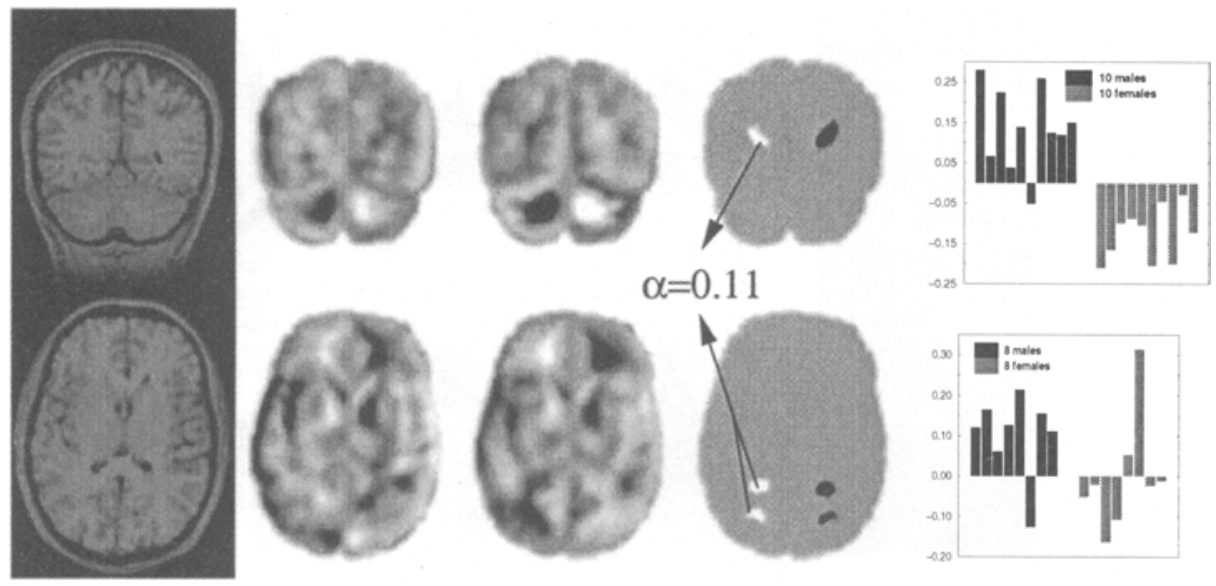

Fig. 5. From left to right: reference subject; average of $d v / v$ im the female learning series; statistical map comparing the 2 populations (coronal and axial views, $l=0.001$ ); $d v / v$ measure on the learning (top) and the validation (bottom) series for the most significant cluster in the left hemisphere.
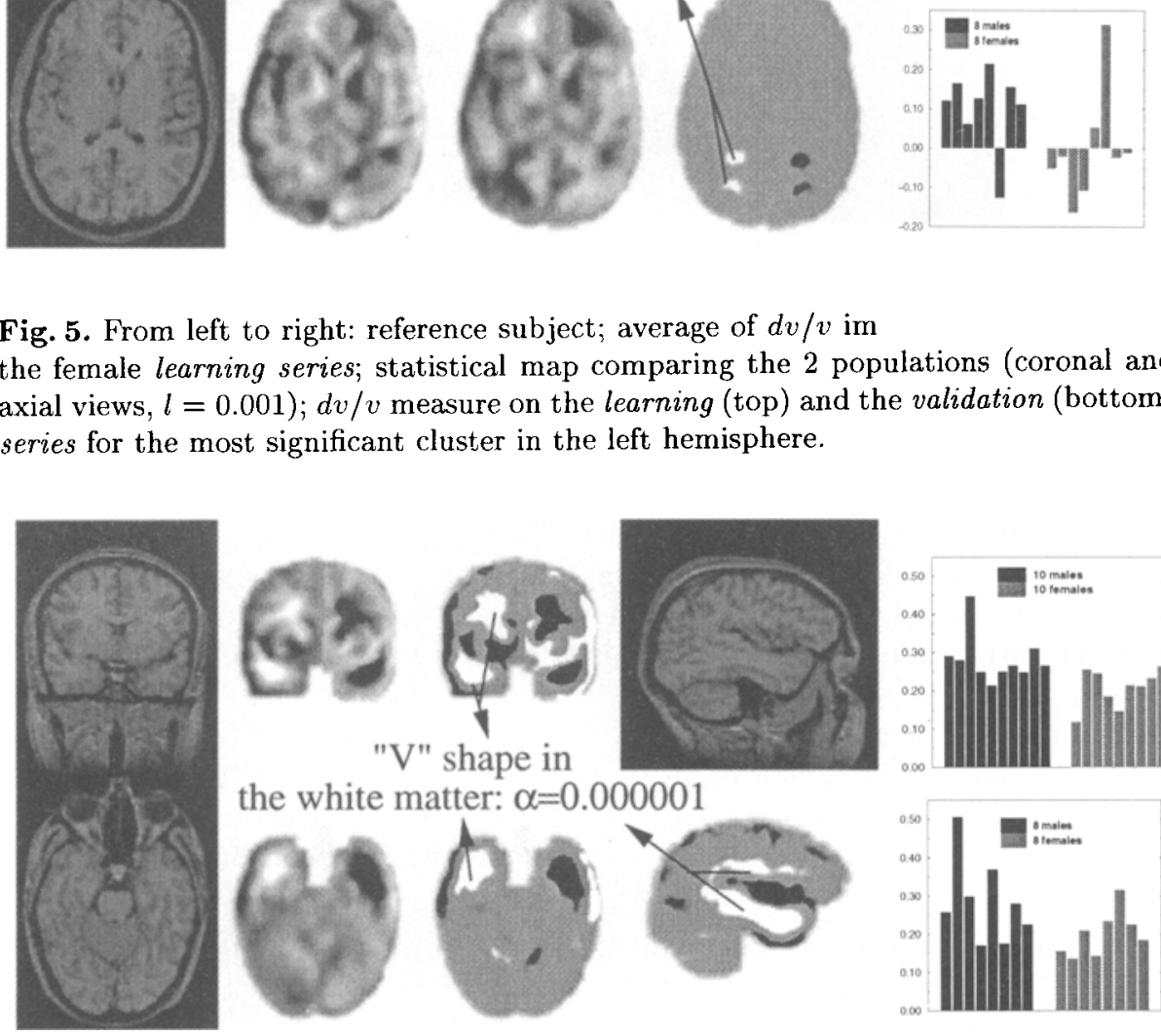

\section{the white matter: $\alpha=0.000001$}
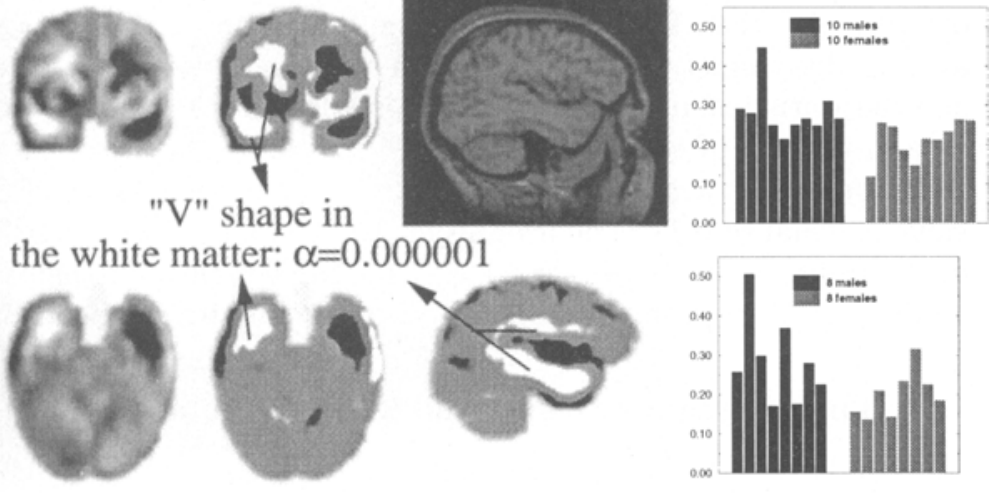

Fig. 6. From left to right: reference subject; average of $d v / v$ images for the whole learning series; statistical map (coronal and axial views, $l=0.001$ ); sagittal view of the reference subject and the map; $d v / v$ measure on the learning (top) and the validation (bottom) series for the most significant cluster in the left hemisphere: the "V-shape".

\section{Conclusion}

We have presented a method to study the dissymmetry of roughly symmetrical objects such as brains. We have shown the usefulness of this method to assess firmly essential results for brain normal anatomy. It was long known that brains in normal subjects were dissymmetrical with always the same laterality, i.e., with 
the right temporal lobe generally larger than the left one. We have shown on a population of 36 normals (18 males and 18 females) that this phenomenon is consistent ( 36 out of 36$)$, with a high level of confidence $(p=0.000001)$. Furthermore, we have been able to refine the shape of this dissymmetric region, which defines a kind of " $V$ " shape, mainly located in the white matter of the brain, with one branch extending in the frontal lobe and the other one in the temporal lobe. The comparison between males and females has lead to the definition of a discriminant area that should be validated on a larger database. We are currently applying the same method on a database of 40 schizophrenic patients and 40 controls within the EC Biomed II BIOMORPH Project.

\section{References}

1. T.J. Crow. Schizophrenia as an anomaly of cerebral asymmetry. In K. Maurer, editor, Imaging of the Brain in Psychiatry and Related Fields, pages 1-17. SpringerVerlag, Berlin Heidelberg, 1993.

2. K.J. Friston, K.J. Worsley, R.S.J. Frackowiak, J.C. Mazziotta, and A.C. Evans. Assessing the Significance of Focal Activations Using their Spatial Extent. Human Brain Mapping, 1:214-220, 1994.

3. Y. Liu, R.T. Collins, and W.E. Rothfus. Automatic bilateral symmetry (midsagittal) plane extraction from pathological 3D neuroradiological images. In SPIE, International Symposium on Medical Imaging, San-Diego, CA, February 1998.

4. P. Marais, R. Guillemaud, M. Sakuma, A. Zisserman, and M. Brady. Visualising Cerebral Asymmetry. In Höhne, K.H. and Kikinis, R., editor, Visualization in Biomedical Computing, volume 1131 of Lecture Notes in Computer Science, pages 411-416, Hamburg (Germany), September 1996. Springer.

5. S. Minoshima, K.L. Berger, K.S. Lee, and M.A. Mintun. An automated method for rotational correction and centering of three-dimensional brain images. $J \mathrm{Nucl}$ Med, 33(8):1579-1585, August 1992.

6. B.A. Shaywitz, S.E. Shaywitz, K.R. Pugh, R.T. Constable, P. Skudlarski, R.K. Fulbright, R.A. Bronen, J.M. Fletcher, D.P. Shankweller, L. Katz, and J.C. Gore. Sex differences in the functional organization of the brain for language. Nature, 373:607-609, February 1995.

7. SPM96. http://www.fil.ion.bpmf.ac.uk/spm/.

8. J-P Thirion. Fast non-rigid matching of $3 \mathrm{D}$ medical images. In Medical Robotics and Computer Aided Surgery (MRCAS'95), pages 47-54, Baltimore, November 1995. Electronic version: http://www.inria.fr/RRRT/RR-2547.html.

9. J-P Thirion and $\mathrm{G}$. Calmon. Measuring lesion growth from 3D medical images. In Nonrigid and Articulated Motion Workshop (NAM'g7), Puerto Rico, June 1997. IEEE. Electronic version : http://www.inria.fr/RRRT/RR-3101.html.

10. J.-P. Thirion, S. Prima, G. Subsol, and N. Roberts. Statistical Analysis of Normal and Abnormal Dissymmetry in Volumetric Medical Images. In IEEE Workshop on Biomedical Image Analysis, WBIA'98, Santa Barbara, USA, June 1998. Electronic version : http://www.inria.fr/RRRT/RR-3178.html.

11. J-P Thirion, G. Subsol, and D. Dean. Cross validation of three inter-patients matching methods. In $V B C$, volume 1131 of Lecture Notes in Computer Science, pages 327-336, Hamburg, Germany, September 1996.

12. S.F. Witelson and D.L. Kigar. Sylvian fissure morphology and asymmetry in men and women: Bilateral differences in relation to handedness in men. The Journal of Comparative Neurology, 323:326-340, 1992. 